Nauki o komunikacji społecznej i mediach

Zeszyty Naukowe KUL 61 (2018), nr 3 (243)

\title{
II messaggio missionario della Radio Vaticana di fronte ai cambiamenti nei media della Santa Sede
}

\begin{abstract}
$\mathrm{D}_{\mathrm{i}}$ a diversi anni continua il processo di ristrutturazione dei media vaticani, iniziato da Papa Francesco. Dal 27 giugno 2015, quando con la Lettera Apostolica in forma di Motu Proprio il Santo Padre ha creato la Segreteria per la Comunicazione ${ }^{1}$, questo processo ha ricevuto una nuova dimensione: ha avviato specifici cambiamenti nelle strutture e nei programmi di tutte le entità di media che hanno operato in Vaticano fino ad ora ${ }^{2}$. Non c'è dubbio che la riforma, che è stata avviata e programmata per alcuni anni, cambierà radicalmente la strategia mediatica della Santa Sede e il ruolo e l'importanza di ogni singola entità di questo consorzio mediatico centralizzato ${ }^{3}$.

Tuttavia, qualsiasi processo di ristrutturazione che riguarda la razionalizzazione del funzionamento dell'istituzione, i potenziali risparmi, le azioni correttive e l'attuazione degli obiettivi adottati, può anche avere effetti collaterali e conseguenze non sempre prevedibili. Dubbi e domande, ovviamente, emergono anche nell'ambiente della riforma dei media vaticani.
\end{abstract}

\footnotetext{
* Leszek Gęsiak SJ - Accademia Ignaziana di Cracovia; e-mail: leszek.gesiak@ignatianum.edu.pl

${ }^{1}$ Francesco PP, «Litterae Apostolicae Motu Proprio Datae Quibus Secretaria pro Communicatione conditur", Acta Apostolicae Sedis, CVII (2015) 591-592.

2 Secondo la riforma strutturale, al nuovo dicastero appartengono tutte le entità associate a media e attività editoriali del Vaticano ampiamente comprese e finora autonome: Pontificio Consiglio delle Comunicazioni Sociali; Sala Stampa della Santa Sede; Servizio Internet Vaticano; Radio Vaticana; Centro Televisivo Vaticano; L'Osservatore Romano; Tipografia Vaticana; Servizio Fotografico; Libreria Editrice Vaticana; cf. Francesco PP, «Litterae Apostolicae» (cf. nt. 1), 591-592.

${ }^{3}$ Ho descritto in modo più dettagliato il processo di riforma dei media vaticana in: L. Gęsiak, «Reform of the Vatican media, a difficult search for a new path», The Person and the Challenges, 7 (2017) 5-22.
} 
Uno dei problemi sollevati è la loro funzione missionaria. I dubbi appaiono a vari livelli, ma uno dei più articolati è la discussione sull'impatto della radio del Papa sulle cosiddette «aree missionarie».

\section{Media Vaticani: cambiamento di concetto}

La Radio Vaticana che funziona dal 1931, dal 1 gennaio 2017, non è più un'unità autonoma. Ora è subordinata alla Segreteria per la Comunicazione dal punto di vista organizzativo, decisivo e della programmazione ${ }^{4}$. Ma questo non è l'unico cambiamento; l'intero processo della riforma riguarda principalmente il concetto del funzionamento del sistema dei media vaticani.

Come ha ripetutamente sottolineato il primo prefetto della Segreteria per la Comunicazione, Mons. Dario Edoardo Viganò (che ha svolto questa funzione dal 27 giugno 2015 al 21 marzo 2018), nell'era dei media elettronici, l'attenzione principale sarà rivolta all'apprezzamento di Internet e del social network che, nell'area dei media vaticani, ora vengono sviluppati in modo molto dinamico. In un'intervista al Vatican Insider, Viganò ha detto:

II rafforzamento della nostra presenza sui social network costituisce uno degli effetti del grande processo di riforma dei media vaticani in corso di completamento. Ed è certamente un effetto positivo raggiunto grazie all'intenso impegno dei nostri giornalisti e dei nostri tecnici. Come operatori della comunicazione, secondo la logica della Chiesa in uscita, tutti siamo chiamati a stare in mezzo alla gente. Oggi questo vuol dire abitare le reti sociali Internet con convinzione e responsabilità. Quindi deve essere molto chiara la nostra prospettiva che esige di mettere al centro la persona, la relazione, la cultura dell' incontro e, solo in ultima battuta, la tecnologia 5 .

Questa prospettiva sembra ovvia dal punto di vista delle società occidentali. Lassenza di una presenza significativa della Chiesa e della voce del Papa su Internet, e in particolare sui social media, sarebbe nel mondo di oggi un enorme errore e una negligenza. La difficoltà, tuttavia, è che il punto di vista del mondo occidentale non è l'unico. Se il Papa incoraggia così spesso l'«andare al confine» e il prendersi cura dei respinti, dei perseguitati e degli emarginati, allora non si può trascurare che il messaggio del Vangelo deve raggiungere tutte le persone sulla terra e, quindi, anche coloro che vivono nella povertà o nell'arretratezza

${ }^{4}$ Cf. Radio Vaticana, «Zmiany w watykańskich strukturach medialnych», http://pl.radiovaticana.va/news/2016/12/31/zmiany_w_watyka\%C5\%84skich_strukturach_medialnych/1282859 (accesso 2.02.2018).

${ }^{5}$ Cf. Vatican Insider, «Vaticano, 4 milioni di utenti sui social della Santa Sede», http://www. lastampa.it/2018/01/09/vaticaninsider/ita/news/vaticano-milioni-di-utenti-sui-social-della-santa-sede-rqKXcuVLS9OfNRcWEmloDK/pagina.html (accesso 2.02.2018). 
economica e non sono in grado di affrontare le sfide tecnologiche che cambiano continuamente e dinamicamente il mondo moderno.

Questo problema è emerso nella discussione sulla possibilità di limitare le forme tradizionali di comunicazione della Radio Vaticana. Si tratta principalmente di rinunciare o ridurre le trasmissioni di programmi radiofonici sulle onde corte e medie che oggi sono utilizzate sempre meno. Questi sistemi dovevano essere sostituiti dalle trasmissioni via Internet e dalle ritrasmissioni nelle stazioni radio locali, dove il segnale radiofonico sarebbe arrivato via Internet o, eventualmente, via satellite.

Una soluzione del genere, abbastanza logica e ovvia dal punto di vista del mondo occidentale, non corrisponde pienamente alle attese dei paesi poveri, in particolare i cosiddetti paesi di missione in Africa. Nella discussione in corso su questa materia, i vescovi di questo continente hanno espresso i loro dubbi, in particolare nella lettera speciale del Consiglio Permanente del Simposio delle Conferenze Episcopali d'Africa e del Madagascar (SECAM), riunitosi nella capitale del Ghana, Accra, il 10 marzo 2017. I gerarchi hanno espresso preoccupazione circa la proposta di porre fine alle trasmissioni della Radio Vaticana sulle onde corte.

Dopo questo incontro, hanno annunciato in un comunicato ufficiale: «La radio ha offerto a milioni di africani l'opportunità di ascoltare il Santo Padre e condividere le preoccupazioni e la missione della Chiesa ${ }^{6}$. I vescovi hanno espresso l'apprezzamento e la gratitudine alla stazione del Papa per il suo ruolo nell'evangelizzazione dell'Africa, catechizzazione e sviluppo spirituale degli abitanti del continente. Hanno sottolineato anche che la Radio Vaticana è sempre stata una fonte affidabile di informazioni dalla Chiesa universale e un veloce canale per avere notizie dell'Africa e del resto del mondo. Hanno notato i limiti che potrebbero essere generati dalla decisione del Segretariato per la Comunicazione di limitare le tradizionali forme di comunicazione. "Riconoscendo che i servizi della Radio Vaticana devono essere ricevuti attraverso Internet, vediamo anche il fatto che molti africani semplicemente non hanno i mezzi o la tecnologia per tali servizi» - ha scritto il Consiglio Permanente di SECAM ${ }^{7}$. I vescovi hanno sottolineato che la situazione può causare delle difficoltà. Per questo chiedono di ripristinare la trasmissione sulle onde corte per proseguire con successo la missione evangelizzatrice del continente africano.

${ }^{6}$ SECAM's Standing Committee "expressed concern about the recent shutdown of the shortwave services of the radio, which afforded millions of Africans the opportunity to hear (the) Holy Father and share in the Church's concerns and mission»; cf. O. Don Bosco, «Bishops in Africa Request for Restoration of Vatican Radio Shortwave Services», https://secam.org/bishops-in-africa-request-forrestoration-of-vatican-radio-shortwave-services/ (accesso 17.02.2018).

7 While recognizing that Vatican Radio services can still be received through the Internet, the fact that many Africans simply do not have the means or the technology to enjoy such services; $\mathrm{cf}$. O. Don Bosco, «Bishops in Africa», (cf. nt. 6). 
La lettera dei vescovi africani ha ricevuto ampia diffusione in ambienti missionari, specialmente in Africa, ma non solo ${ }^{8}$. Alcuni media hanno sottolineato che, mentre la Radio Vaticana vuole abbandonare le onde corte, altre stazioni radio stanno facendo il contrario. Un esempio sono i grandi consorzi di media, come la britannica BBC (British Broadcasting Corporation) o la giapponese NHK (Nippon Hōsō Kyōkai). Il primo ha aumentato il budget per le emissioni sulle onde corte da 56 a quasi 85 milioni di sterline, principalmente per essere presente in Russia, Corea del Nord, Medio Oriente e, precisamente, in Africa9.

\section{La Chiesa e la sua priorità missionaria}

«La Chiesa durante il suo pellegrinaggio sulla terra è per sua natura missionaria, in quanto è dalla missione del Figlio e dalla missione dello Spirito Santo che essa, secondo il piano di Dio Padre, deriva la propria origine ${ }^{10}$. Queste parole del Concilio Vaticano II, tratte dal decreto Ad gentes, sottolineano inequivocabilmente che l'evangelizzazione, e quindi la possibilità che la Buona Novella raggiunga tutti i popoli della Terra, è una delle priorità assolute della Chiesa. «Inviata per mandato divino alle genti per essere "sacramento universale di salvezza" la Chiesa, rispondendo a un tempo alle esigenze più profonde della sua cattolicità ed all'ordine specifico del suo fondatore, si sforza di portare l'annuncio del Vangelo a tutti gli uomini ${ }^{11}$ - aggiunge lo stesso decreto. Ciò significa che dovrebbero essere usati tutti i mezzi, compresi i social media, se possono servire a questa missione principale.

Molti Papi hanno confermato questa consapevolezza nel loro insegnamento. Tuttavia questo è stato particolarmente sottolineato da Giovanni Paolo II. Il suo pontificato, durato 26 anni, si è collocato al tempo dello sviluppo dei mass media e che egli ha saputo usare molto efficacemente, specialmente come

${ }^{8}$ Guarda per esempio: Agence Fides, «Appel du Comité permanent du SCEAM en faveur de la reprise des transmissions en ondes courtes de Radio Vatican», http://www.fides.org/fr/news/61901AFRIQUE_GHANA_Appel_du_Comite_permanent_du_SCEAM_en_faveur_de_la_reprise_des_ transmissions_en_ondes_courtes_de_Radio_Vatican\#.WMKPpVXhD0M (accesso 17.02.2018); guarda anche: Portail catholique suisse, «Les évêques africains demandent que Radio Vatican continue à émettre sur ondes courtes», https://www.cath.ch/newsf/eveques-africains-demandentradio-vatican-continue-a-emettre-ondes-courtes (accesso 17.02.2018).

9 Cf. S. Magister, «Radio Vaticana. Fine delle onde corte, un pessimo affare», L'Espresso, (3.03.2017), http://magister.blogautore.espresso.repubblica.it/2017/03/03/radio-vaticana-fine-delle-onde-corte-un-pessimo-affare (accesso 17.02.2018).

10 Concilio Vaticano II, «Decreto sull'attività missionaria delle Chiesa Ad gentes», http://www. vatican.va/archive/hist_councils/ii_vatican_council/documents/vat-ii_decree_19651207_ad-gentes_it.html, n. 2 (accesso 24.02.2018).

${ }^{11}$ Concilio Vaticano II, «Decreto sull'attività missionaria», (cf. nt. 10) n. 1. 
Papa-Pellegrino nei 104 viaggi fuori Italia, mentre visitava circa 900 città ${ }^{12}$. Dal primo giorno del suo compito, è stato accompagnato da una macchina fotografica e da un microfono che sono diventati un elemento inseparabile del suo servizio. I media e, in particolare, la Radio Vaticana che ha giocato il ruolo centrale nella comunicazione in questo ministero, sono diventati un elemento inseparabile del suo ministero pastorale. Anche la stessa emittente ha superato lo standard normale di questo tipo d'istituzione, diventando un mezzo mobile per trasmettere il messaggio del Papa a tutto il mondo e da tutto il mondo. La Radio Vaticana non solo ha raggiunto le case dei fedeli attraverso le onde inviate da Roma. Insieme alle sue attrezzature, giornalisti, redattori e tecnici hanno costruito i centri di trasmissione in tutti i paesi raggiunti dal Papa per annunciare il messaggio del Vangelo.

Il 7 dicembre 1990 Giovanni Paolo II, in occasione del venticinquesimo anniversario del decreto del Consiglio Vaticano II Ad Gentes, ha pubblicato l'enciclica Redemptoris Missio ${ }^{13}$, dedicata alle missioni. Questo documento ha sottolineato l'importanza dell'evangelizzazione nel mondo moderno, con particolare attenzione ai cosiddetti «paesi di missione». E, quindi, è comprensibile che in questo documento il Papa abbia ricordato che il primo contemporaneo «areopago» è il mondo dei media, perché unisce l'umanità e la rende come «un villaggio globale» ${ }^{14}$.

Le nuove generazioni soprattutto crescono in modo condizionato da essi. Forse è stato un po' trascurato questo areopago: si privilegiano generalmente altri strumenti per l'annunzio evangelico e per la formazione, mentre i mass media sono lasciati all'iniziativa di singoli o di piccoli gruppi ed entrano nella programmazione pastorale in linea secondaria. L'impegno nei mass media, tuttavia, non ha solo lo scopo di moltiplicare l'annunzio: si tratta di un fatto più profondo, perché l'evangelizzazione stessa della cultura moderna dipende in gran parte dal loro influsso. Non basta, quindi, usarli per diffondere il messaggio cristiano e Magistero della chiesa, ma occorre integrare il messaggio stesso in questa «nuova cultura» creata dalla comunicazione moderna. Ė un problema complesso, poiché questa cultura nasce, prima ancora che dai contenuti, dal fatto stesso che esistono nuovi modi di comunicare con nuovi linguaggi, nuove tecniche e nuovi atteggiamenti psicologici ${ }^{15}$.

I media, secondo la valutazione offerta, non solo sono uno strumento privilegiato per l'evangelizzazione, essi sono cruciali per l'evangelizzazione della

${ }^{12}$ Cf. «Pielgrzymki», http://www.janpawel2.pl/jan-pawe-ii/pielgrzymkil (accesso 3.02.2018).

${ }_{13}$ Giovanni Paolo II, «Lettera Enciclica Redemptoris Missio», http://w2.vatican.va/content/ john-paul-ii/it/encyclicals/documents/hf_jp-ii_enc_07121990_redemptoris-missio.html (accesso 24.02.2018).

${ }^{14}$ Giovanni Paolo II, «Lettera Enciclica» (cf. nt. 13) n. 37c .

15 Giovanni Paolo II, «Lettera Enciclica» (cf. nt. 13). 
cultura contemporanea. E questo significa che il messaggio cristiano, cuore dell'evangelizzazione, dovrebbe essere incluso nella cultura creata dai mezzi di comunicazione di massa.

Vale la pena prestare attenzione anche a una diversa citazione di Giovanni Paolo II in questa materia. Cinque anni più tardi, nell'Esortazione apostolica post-sinodale Ecclesia in Africa ${ }^{16}$, ha ricordato che «i moderni mass-media non costituiscono soltanto strumenti di comunicazione; sono anche un mondo da evangelizzare. Circa i messaggi da essi trasmessi, bisogna assicurarsi che vi si propongano il bene, il vero e il bello» ${ }^{17}$. Se non sono usati correttamente, o semplicemente il loro uso è interrotto, può causare seri danni. Questi timori, soprattutto per quanto riguarda la diffusione della pornografia e della violenza, sono state sollevate dai vescovi africani durante il Sinodo. Pertanto, anche «ogni cristiano deve preoccuparsi che i mezzi di comunicazione siano veicolo di evangelizzazione. Ma il cristiano che opera come professionista in questo settore ha un suo ruolo speciale da svolgere ${ }^{18}$. Si fa riferimento ai giornalisti, ma anche ai tecnici e agli amministratori dei media che, per svolgere adeguatamente il loro compito, dovrebbero essere dotati di una sana formazione umana, religiosa e spirituale ${ }^{19}$.

Anche allora, il Papa ha ricordato che la Chiesa, nonostante utilizzi i media nel suo lavoro, sia tradizionali sia moderni, deve usarli per proclamare il messaggio della salvezza. Tuttavia, nel caso della Chiesa in Africa, l'accesso a questi mezzi può essere più difficile ${ }^{20}$. Questa è un'osservazione molto importante. Dimostra che, a causa della diversità nell'accesso alle nuove tecnologie, nel caso dei paesi del terzo mondo e delle società meno sviluppate, abbiamo spesso situazioni seriamente arretrate. Le nuove tecnologie sono semplicemente costose per le società più povere che combattono spesso contro la fame o vivono in un contesto di guerre e violenze. Il rallentamento economico e le sproporzioni sociali, evidenti nel mondo moderno, determinano anche la mancanza di accesso alle nuove tecnologie. Giovanni Paolo II che nell'esortazione ha chiesto la rimozione di tutte le restrizioni ingiuste nel campo dell'accesso ai media moderni, ha sottolineato che tutti i mezzi di comunicazione sociale, senza eccezioni, privati o pubblici, dovrebbero servire le persone ${ }^{21}$.

\footnotetext{
${ }^{16}$ Giovanni Paolo II, «Esortazione Apostolica Post-Sinodale Ecclesia in Africa», http://w2.vatican. va/content/john-paul-ii/it/apost_exhortations/documents/hf_jp-ii_exh_14091995_ecclesia-in-africa. html (accesso 24.02.2018).

17 Giovanni Paolo II, «Esortazione Apostolica» (cf. nt. 16).

18 Giovanni Paolo II, «Esortazione Apostolica» (cf. nt. 16) n. 124.

19 Cf. Giovanni Paolo II, «Esortazione Apostolica» (cf. nt. 16) n. 124.

20 Cf. Giovanni Paolo II, «Esortazione Apostolica» (cf. nt. 16) n. 125.

${ }^{21}$ Cf. Giovanni Paolo II, «Esortazione Apostolica» (cf. nt. 16) n. 125.
} 
Inoltre, il Papa, sottolineando che l'influenza dei media non è soggetta ad alcuna restrizione spaziale nel mondo di oggi, ha incoraggiato lo stretto coordinamento in questo campo così da consentire un'efficace cooperazione a tutti i livelli: da diocesano a globale. «In Africa, la Chiesa ha molto bisogno della solidarietà delle Chiese sorelle dei Paesi più ricchi e più avanzati dal punto di vista tecnologico» - ha sottolineato in Ecclesia in Africa ${ }^{22}$.

\section{L'universalità missionaria della Chiesa nella Radio Vaticana}

Tenendo presente l'analisi precedente, vale la pena chiedersi come la Radio Vaticana ha risolto il problema della sua missione nel contesto della Chiesa universale. Per la sua stessa natura, dall'inizio, la Radio era una stazione multilingue e multiculturale. Naturalmente questo era in sintonia con l'universalità del messaggio del Vangelo e con il ministero dei Successori di San Pietro. La radio del Papa, costruita da Pio XI, aveva, tra gli altri, lo scopo di inviare questo messaggio universale del Vangelo a tutti i fedeli, indipendentemente dal loro paese, dalla lingua che usavano o dal sistema politico in cui vivevano.

Il 12 febbraio 1931, Pio XI inaugurò ufficialmente il lavoro della radio in un edificio costruito per questo scopo nei giardini vaticani ${ }^{23}$. Sin dall'inizio, a causa della specificità della nuova stazione della radio, si è deciso di fare questo lavoro in varie lingue. La prima, il latino, come lingua ufficiale del Vaticano; l'italiano, perché la Radio è un enclave in Italia; il francese, che allora era la lingua della diplomazia, ma anche la lingua con una valenza internazionale ${ }^{24}$. Con il tempo sono cominciati programmi in altre lingue. La scelta delle varie lingue fu fatta perché, grazie alla loro universalità, potevano servire nel lavoro missionario.

Con il tempo, nella Radio Vaticana si sono organizzate le sezioni linguistiche, con attenzione a quelle lingue che sono principalmente utilizzate nelle aree missionarie. Già nel primo anno di esistenza della stazione radio, fu creata la Sezione Francese e, nel 1937, la Sezione Inglese. Negli anni ‘50, in entrambe le sezioni, sono state create le sottosezione dedicate specialmente ai paesi dell'Africa. Nel tempo, queste sono state trasformate in sezioni indipendenti, d'inglese e di francese, per l'Africa ${ }^{25}$. Era chiaro che la preparazione di programmi per regioni del mondo culturalmente diverse, anche se usano la stessa lingua, richiede una specifica edizione. Per quanto riguarda l'inglese, con gli anni, è stato deciso

\footnotetext{
${ }^{22}$ Giovanni Paolo II, «Esortazione Apostolica» (cf. nt. 16) n. 126.

23 Cf. F. Bea - A. De Carolis, Ottant'anni della Radio del Papa, 1, Città del Vaticano, 2011, 38.

24 Cf. L. Grzebień, Sekcja Polska Radia Watykańskiego 1938-1955, Rzym, 1990, 51.

${ }^{25}$ Cf. F. Bea - A. De Carolis, Ottant'anni della Radio del Papa, 2, Città del Vaticano, 2011, 143.
} 
di creare una sezione di questa lingua impegnata a preparare i programmi per il circolo culturale asiatico, in particolare per le comunità cattoliche in India. Questo vuol dire che i programmi in inglese, ogni giorno, sono stati preparati in tre diverse sezioni: quella generale, quella per l'Africa e quella per l'Asia.

Un ruolo importante è giocato anche dalla Newsletter della Radio Vaticana. Ė stata redatta per iscritto in diverse lingue. Secondo la lingua e il paese di destinazione, è stata stampata e inviata per posta con diversa frequenza. Dopo la creazione e lo sviluppo di Internet, è stata rilasciata in forma elettronica. La Newsletter è stata preparata, con particolare attenzione alle aree di missione. Questa funzione è stata affidata ai reparti specificamente incentrati sui bisogni missionari: l'Ufficio di Promozione per l'Africa, in francese, portoghese e inglese ${ }^{26}$ e l'Ufficio per la Promozione dell'America Latina, in spagnolo ${ }^{27}$.

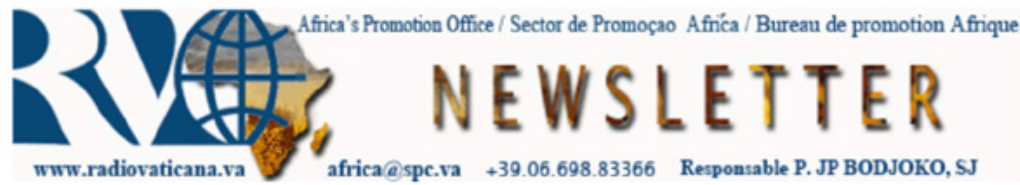

$31 / 01 / 2018$

\section{De nous à vous - From us to you - De nós para vós}

- Catéchèse du Pape (31/01/2018)/Pope's catechesis (31/01/2018)/Catequese do Papa (31/o1/2018)

- VATICANO /RCA: Papa recebe o Presidente Touadéra

- Vatican Radio Communiqué/Communiqué Radio Vatican/Comunicado da Rádio Vaticano

- Avis pour les radios catholiques africaines et les auditeurs de Radio Vatican/ Notice to Catholic Radio Stations in Africa and listeners of Vatican Ra

- Nos fréquences en $\mathrm{KHz}$ sur les Ondes courtes (Heure en Temps universel)/ Our

Fig. 1. Un esempio della prima pagina della Newsletter in francese preparata dall'Ufficio di Promozione per l'Africa della Radio Vaticana dal 31 gennaio 2018; cf. http://fr.radiovaticana.va/archives-newsletter (accesso 3.02.2018).

${ }^{26}$ Cf. Radio Vaticana, Africa's Promotion Office / Sector de promoçáo Africa / Bureau de promotion Afrique, «Newsletter», http://fr.radiovaticana.va/archives-newsletter (accesso 3.02.2018).

${ }^{27}$ Cf. Radio Vaticana, Oficina promoción América Latina, «Boletín electrónico semanal», http:// es.radiovaticana.va/boletin-america-latina (acceso 3.02.2018). 


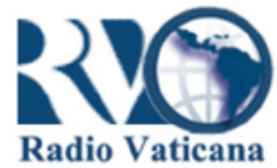

\section{Para Latinoamérica Alternativa Multimedia}

- Cadena de Amistad • Proqramación en Español • Centro Televisivo Vaticano • Si no desea recibir este boletín semanal escriba a newsletter.latam.office@vatiradio.va Sumario del 14/10/2017

\section{Pedro en diálogo con las culturas}

- El Papa: "Con su oración y compromiso personal colaboran con la misión del Sucesor de Pedro"

- Catequesis del Papa: “El cristiano constructor de paz, en espera vigilante del regreso de Jesús"

\section{Hacia el encuentro}

Fig. 2. Un esempio della prima pagina della Newsletter in spagnolo preparato dall'Ufficio per la Promozione dell'America Latina della Radio Vaticana del 14 ottobre 2017; cf. http://es.radiovaticana. va/boletin-america-latina (accesso 3.02.2018).

Ma le aree missionarie non sono solo l'Africa o l'America Latina, perciò si sono definite nuove sezioni e sono state introdotte nuove lingue nel lavoro quotidiano. Nel 1961, la Radio Vaticana aveva già un numero significativo di sezioni indipendenti. Per la maggior parte, si trattava di sezioni che preparavano i programmi per l'Europa, in particolare l'Europa dell'Est. C'erano anche due sezioni per l'America Latina: la brasiliana e la spagnola. Le sezioni per l'Africa sono: l'etiope, la francese, l'inglese e la portoghese, mentre per i paesi asiatici i programmi sono stati fatti in arabo, cinese, giapponese e inglese ${ }^{28}$. All'inizio del 2018, i siti web della multiculturale Radio Vaticana sono stati sviluppati in 39 lingue, di cui una parte significativa sono le lingue utilizzate nelle aree di missione.

${ }^{28}$ Cf. L’Osservatore Romano, 13-14 febbraio 1961, citazione da: F. Bea, Qui Radio Vaticana. Mezzo secolo della Radio del Papa, Città del Vaticano, 1981, 200-201. 


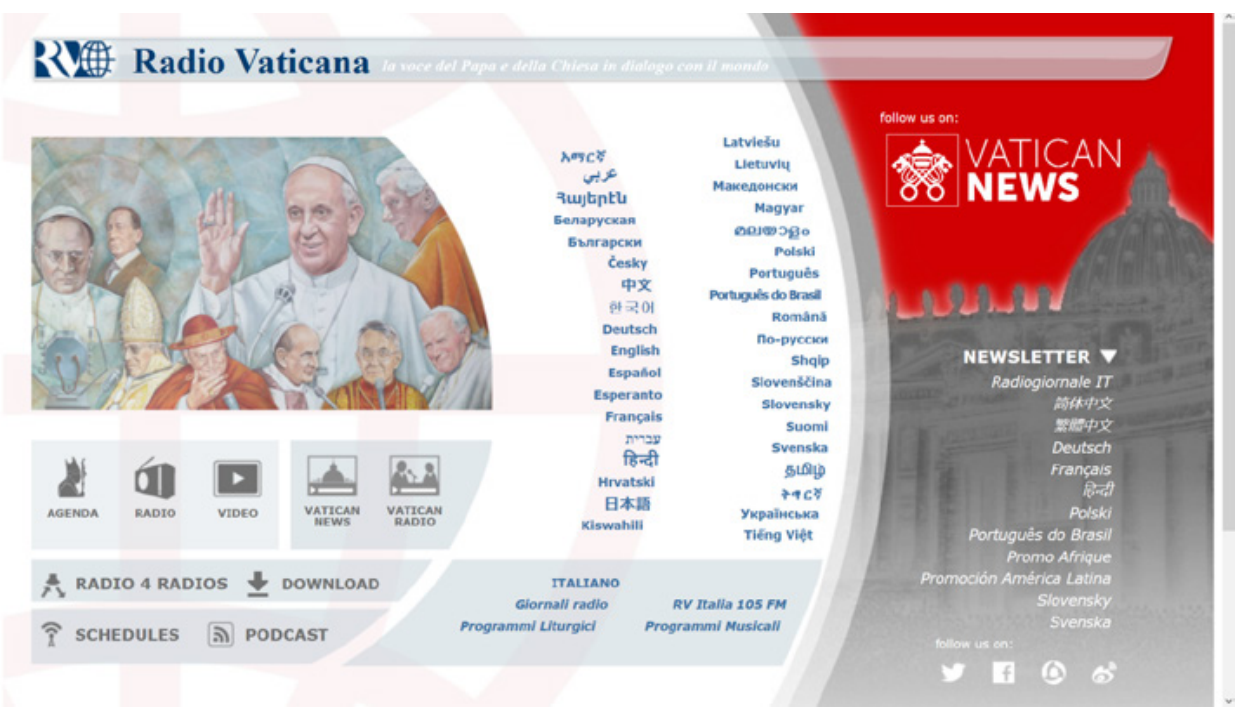

Fig. 3. La pagina principale del sito web della Radio Vaticana http://www.radiovaticana.va/ (accesso 3.02.2018) con i collegamenti alle pagine in 39 lingue.

\section{II ruolo missionario della Chiesa nella riforma dei media vaticani}

Come già detto, la riforma dei media vaticani consiste, da una parte, nell'integrazione della politica mediatica del Vaticano e, dall'altra, dall'adattamento alle esigenze del mondo moderno. La Radio Vaticana è sempre stata nel mondo un'avanguardia tecnologica per questo tipo di opere. Con le sue soluzioni moderne spesso ha superato nel tempo altre emittenti e società di media. Il semplice fatto che il Papa abbia accettato di costruire la stazione in Vaticano, confidando nella guida dell'inventore Guglielmo Marconi, dimostra che questo è stato un lavoro pionieristico. Così l'ha descritto il cardinale Carlo Confalonieri, testimone di quei primi eventi:

Pio XI si accinse alla realizzazione della Sua Radio con la sua solita decisa risolutezza, e Guglielmo Marconi si prestò con dedizione ammirabile. Ha seguito di un'udienza pontificia (11 giugno 1929, a solo quattro giorni dallo scambio delle ratifiche dei Patti Lateranensi), il grande inventore fu accompagnato dallo scrivente nei Giardini Vaticani per il primo sopraluogo. La modestia di Marconi impressionava e edificava. È proprio vero che il genio non abbisogna di pose: sono i piccoli uomini che si pavoneggiano e si danno le arie. Qualche minuto dopo giunse sul posto il neomarchese avv. Francesco Pacelli, uno dei principali collaboratori dei Patti, che, nel frattempo, era stato fatto avvertire. Il lavori d'impianto furono presto iniziati e condotti alacremente a termine ${ }^{29}$.

${ }^{29}$ Cf. C. Confalonieri, Pio XI visto da vicino, Torino, 1957, 147-148. 
Certamente, la Radio Vaticana ha cercato di rimanere nell'avanguardia radiofonica per tutta la sua storia. Ha modernizzato e adattato le sue attrezzature alle nuove esigenze tecnologiche, ponendo sempre l'attenzione sull' importanza del suo messaggio missionario. Un esempio è stata l'apertura, il 26 ottobre 1957, di uno dei più moderni centri di trasmissione a Santa Maria di Galleria, a circa $30 \mathrm{~km}$ da Roma, costruito in pochi anni ${ }^{30}$. Questo centro ha offerto la possibilità di installare antenne ad alta potenza che hanno permesso la trasmissione dei programmi direttamente dalla stazione ad una distanza molto lunga, utilizzando specialmente le onde corte.

Nel 1978, anno della scelta di Karol Wojtyla come Papa, nel centro di radiodiffusione di Santa Maria di Galleria fu completata l'installazione di un grande sistema di emissione con una potenza di $500 \mathrm{~kW}$ su onde corte. Questo sistema era correlato con un'antenna rotante in cornici alte $79 \mathrm{~m}$. Nello stesso anno, fu aperta anche una seconda antenna rotante con un'altezza di $106 \mathrm{~m}$; all'epoca era una delle più grandi al mondo del suo genere ${ }^{31}$. Grazie a tali apparecchi e grazie ai contratti firmati con diversi centri di trasmissione nel mondo, il segnale dal Vaticano ha potuto facilmente raggiungere il mondo intero. Ciò significava che, alla massima distanza dalla civiltà, in ogni angolo del globo, dove non c'erano infrastrutture tecniche, dove non era possibile fornire assistenza pastorale e dove, a volte, nessun sacerdote poteva fisicamente esserci, con l'aiuto di una minuscola radio che riceveva onde corte, con un semplice generatore manuale, la Chiesa poteva continuare la sua missione evangelizzatrice.

Introducendo i cambiamenti nelle strutture e nel funzionamento dei media vaticani e sottolineando uno sviluppo tecnologico, soprattutto in relazione ad Internet e ai social media, sono naturali alcune perplessità. In questo processo, il metodo tradizionale di evangelizzazione radiofonica potrebbe cadere. Le prime riduzioni si sono svolte già nel 2012. L'1 luglio, la stazione del Papa ha rinunciato a trasmettere sulle onde medie e onde corte verso l'Europa ed entrambe le Americhe. Lallora direttore generale della Radio Vaticana, p. Federico Lombardi SJ, sosteneva che era Internet che obbligava a questo cambio. Era necessario il passaggio dalla radio, che produceva principalmente i programmi sonori classici, a una gamma più ampia di prodotti mediatici. Assicurava che la missione principale della radio non sarebbe cambiata.

Cerchiamo di seguire questa evoluzione e l'abbiamo fatto con molta consapevolezza, proprio prevedendo che un certo tipo di tecnologia poteva tramontare o perlomeno essere meno necessaria e che quindi dovevamo concentrare l'attenzione sul centro della nostra missione, che è comunicare il Vangelo, il messaggio della Chiesa nel mondo di oggi, il servizio che il Papa svolge al mondo di oggi e comunicarlo il più diffusamente possibile con lingue diverse, ai diversi

${ }^{30}$ F. Bea, Qui Radio Vaticana (cf. nt. 28), 1981, 188-202.

${ }^{31}$ Cf. F. Bea - A. De Carolis, Ottant'anni, 2, (cf. nt. 25) 26. 
popoli del mondo. Questo è il nucleo della nostra missione, ma con quali tecniche, attraverso quali vie questa missione debba realizzarsi è aspetto che naturalmente può cambiare - ha detto padre Lombardi ${ }^{32}$.

\section{Il futuro e le prospettive}

Il processo di cambiamento della Radio Vaticana, iniziato nel 2012, continua. Il sistema di comunicazione dei media vaticani si sta modificando, le nuove tecnologie stanno gradualmente sostituendo quelle vecchie. Alcune delle antenne nel centro di Santa Maria di Galleria sono già spente. La radio multilingue della Santa Sede, i programmi radiofonici in quasi 40 lingue, sono ora disponibili a Roma capitale sulla piattaforma digitale $\mathrm{DAB}+\mathrm{e}$ via Internet. Il nucleo del nuovo assetto è il nuovo portale Vatican News. In questo periodo, le nuove disposizioni sono in fase di lancio in più lingue nella versione beta ${ }^{33}$. Questo portale integra tutte le entità mediatiche esistenti in Vaticano.

Avendo dunque un semplice smartphone con accesso a internet, si può facilmente avere un'idea delle attività attuali del Papa. Si può vedere in diretta o on-demand la copertura completa dei viaggi del Papa o le udienze generali, seguire gli eventi in Vaticano e gli affari più importanti della Chiesa universale e del mondo. Bisogna sperare che questi cambiamenti tecnologici e strutturali dei media vaticani sostengano l'accesso a questi materiali anche da parte delle comunità missionarie della savana e del deserto, lontane dai grandi centri urbani, dove Internet è un'opportunità che non appartiene a tutti. Una soluzione è quella di trasmettere il segnale sonoro al numero più alto di emittenti locali, in modo che il messaggio del Papa possa facilmente raggiungere i fedeli anche nei luoghi dove continuano le guerre e dove i cristiani sono perseguitati.

Le tecnologie moderne sono, naturalmente, la risposta alle sfide del mondo di oggi e la Chiesa deve rispondere loro come da due mille anni risponde alla sfida missionaria di proclamare il Vangelo a tutte le persone sulla terra, specialmente quelle perseguitate, emarginate e rifiutate dal mondo della prosperità e del potere. Questa è anche la volontà di Papa Francesco. Il Santo Padre riconosce l'ovvia necessità di riformare e adattare i media vaticani ai tempi moderni e, nel contempo, chiede di perseverare nella direzione più importante della Chiesa: la sua missione evangelizzatrice. L'ha ricordato nel suo discorso ai partecipanti della prima Sessione Plenaria della Segretaria per la Comunicazione, nel maggio 2017:

${ }^{32}$ A. De Carolis, «Padre Lombardi sulle novità della Radio Vaticana: le tecnologie tramontano, la missione resta», Radiogiornale. Sommario (13.06.2012), http://it.radiovaticana.va/radiogiornale?data $=24 / 06 / 2012$ (accesso 24.02.2018).

${ }^{33}$ Cf. «Vatican News», http://www.vaticannews.va (accesso 4.05.2018). 
Vi chiedo inoltre che il criterio-guida sia quello apostolico, missionario, con una speciale attenzione alle situazioni di disagio, di povertà, di difficoltà, nella consapevolezza che anche queste oggi vanno affrontate con soluzioni adeguate. Così diventa possibile portare il Vangelo a tutti, valorizzare le risorse umane, senza sostituirsi alla comunicazione delle Chiese locali e, al tempo stesso, sostenendo le comunità ecclesiali che più hanno bisogno ${ }^{34}$.

\section{Bibliografia}

Bea Fernando, Qui Radio Vaticana. Mezzo secolo della Radio del Papa, Città del Vaticano, 1981.

Bea Fernando - De Carolis Alessandro, Ottant'anni della Radio del Papa, 1, 2, Città del Vaticano, 2011. Concilio Vaticano II, «Decreto sull'attività missionaria delle Chiesa Ad gentes», http://www.vatican. va/archive/hist_councils/ii_vatican_council/documents/vat-ii_decree_19651207_ad-gentes_it.html (accesso 24.02.2018).

Confalonieri Carlo, Pio XI visto da vicino, Torino, 1957.

De Carolis Alessandro, «Padre Lombardi sulle novità della Radio Vaticana: le tecnologie tramontano, la missione resta», Radiogiornale. Sommario (13.06.2012), http://it.radiovaticana.va/ radiogiornale?data $=24 / 06 / 2012$ (accesso 24.02.2018).

Don Bosco Onyalla, «Bishops in Africa Request for Restoration of Vatican Radio Shortwave Services», https://secam.org/bishops-in-africa-request-for-restoration-of-vatican-radio-shortwaveservices/ (accesso 17.02.2018).

Francesco, «Discorso del Santo Padre Francesco al partecipanti alla Plenaria della Segreteria per la Comunicazione», http://w2.vatican.va/content/francesco/it/speeches/2017/may/documents/ papa-francesco_20170504_plenaria-segreteria-comunicazione.html (accesso 4.02.2018).

Francesco PP, «Litterae Apostolicae Motu Proprio Datae Quibus Secretaria pro Communicatione conditur», Acta Apostolicae Sedis, CVII (2015) 591-592.

Gęsiak Leszek, «Reform of the Vatican media, a difficult search for a new path», The Person and the Challenges, 7 (2017) 5-22.

Giovanni Paolo II, «Esortazione Apostolica Post-Sinodale Ecclesia in Africa», http://w2.vatican.va/ content/john-paul-ii/it/apost_exhortations/documents/hf_jp-ii_exh_14091995_ecclesia-in-africa.html (accesso 24.02.2018).

Giovanni Paolo II, «Lettera Enciclica Redemptoris Missio», http://w2.vatican.va/content/john-paul-ii/ it/encyclicals/documents/hf_jp-ii_enc_07121990_redemptoris-missio.html (accesso 24.02.2018).

Grzebień Ludwik, Sekcja Polska Radia Watykańskiego 1938-1955, Rzym, 1990.

Magister Sandro, «Radio Vaticana. Fine delle onde corte, un pessimo affare», L'Espresso, (3.03.2017), http://magister.blogautore.espresso.repubblica.it/2017/03/03/radio-vaticana-fine-delle-ondecorte-un-pessimo-affare (accesso 17.02.2018).

\section{Altre fonti Internet}

Agence Fides, «Appel du Comité permanent du SCEAM en faveur de la reprise des transmissions en ondes courtes de Radio Vatican», http://www.fides.org/fr/news/61901-AFRIQUE_GHANA_ Appel_du_Comite_permanent_du_SCEAM_en_faveur_de_la_reprise_des_transmissions_ en_ondes_courtes_de_Radio_Vatican\#.WMKPpVXhD0M (accesso 17.02.2018).

${ }^{34}$ Francesco, «Discorso del Santo Padre Francesco al partecipanti alla Plenaria della Segreteria per la Comunicazione», http://w2.vatican.va/content/francesco/it/speeches/2017/may/documents/ papa-francesco_20170504_plenaria-segreteria-comunicazione.html (accesso 4.02.2018). 
«Pielgrzymki», http://www.janpawel2.pl/jan-pawe-ii/pielgrzymki1 (accesso 3.02.2018).

Portail catholique suisse, «Les évêques africains demandent que Radio Vatican continue à émettre sur ondes courtes», https://www.cath.ch/newsf/eveques-africains-demandent-radio-vaticancontinue-a-emettre-ondes-courtes (accesso 17.02.2018).

Radio Vaticana, Africa's Promotion Office / Sector de promoçáo Africa / Bureau de promotion Afrique, «Newsletter», http://fr.radiovaticana.va/archives-newsletter (accesso 3.02.2018).

Radio Vaticana, La voce del Papa e della Chiesa in dialogo con il mondo, http://www.radiovaticana. $\mathrm{va} /(3.02 .2018)$

Radio Vaticana, Oficina promoción América Latina, «Boletín electrónico semanal», http://es.radiovaticana.va/boletin-america-latina (acceso 3.02.2018).

Radio Vaticana, «Zmiany w watykańskich strukturach medialnych», http://pl.radiovaticana.va/ news/2016/12/31/zmiany_w_watyka\%C5\%84skich_strukturach_medialnych/1282859 (accesso 2.02.2018).

Vatican Insider, «Vaticano, 4 milioni di utenti sui social della Santa Sede», http://www.lastampa. it/2018/01/09/vaticaninsider/ita/news/vaticano-milioni-di-utenti-sui-social-della-santa-sede-rqKXcuVLS9OfNRcWEmloDK/pagina.html (accesso 2.02.2018).

«Vatican News», http://www.vaticannews.va (accesso 4.02.2018).

\section{Riassunto}

Da diversi anni è in atto un processo di ristrutturazione dei media vaticani. La Segreteria per la Comunicazione ha iniziato dei radicali cambiamenti delle strutture e dei programmi di tutte le entità di media che operano in Vaticano, attuando nuove strategie. Una delle sfide che continua ad essere molto viva è la funzione missionaria dei media vaticani, finora svolta dalla Radio Vaticana in modo molto efficace.

Una breve presentazione della riforma mostra le idee della trasformazione in atto. Il punto cruciale è l'apertura ad Internet e ai social media. Infatti, le nuove tecnologie non sempre sono accessibili al «mondo missionario», soprattutto a paesi dell'Africa lontani dalle grandi città. La Radio Vaticana deve trovare una buona risposta alle esigenze di tutti per continuare ad essere un mezzo apostolico missionario significativo.

Parole chiave: Africa, Chiesa, comunicazione, Francesco, Giovanni Paolo II, Internet, media, missione, multiculturale, multilingue, Radio Vaticana, riforma, Santa Sede, social media, tecnologie.

\section{The missionary message of the Vatican Radio in the face of changes in the Holy See's media}

\section{Summary}

The process of restructuring the Vatican media has continued for several years. The Secretariat for Communication has initiated changes in the structures and programs of all media entities that have operated in the Vatican. The reform radically changes the media strategy of the Holy See. One of the challenges is the missionary function of the Vatican media, so far carried out by Vatican Radio in a very effective way. A brief presentation of the reform shows the ideas guiding this transformation. The most important factor is the openness of today's world to the Internet and social media. However, it seems that new technologies are not always fully suitable for the "missionary world", especially in many places in Africa. For this reason it seems important to find a new efficient way 
of proceeding. Retaining these, Vatican Radio should continue to be a very significant factor in the missionary activities of the Church.

Keywords: Africa, Church, communication, Francis, Holy See, Internet, John Paul II, media, mission, multicultural, multilingual, reform, social media, technologies, Vatican Radio. 